\title{
Untranslated Element in Neurofilament mRNA Has Neuropathic Effect on Motor Neurons of Transgenic Mice
}

\author{
Zhenying Nie, Junhua Wu, Jinbin Zhai, Hong Lin, Weiwen Ge, William W. Schlaepfer, and \\ Rafaela Cañete-Soler
}

Division of Neuropathology, University of Pennsylvania Medical School, Philadelphia, Pennsylvania 19104

\begin{abstract}
Studies of experimental motor neuron degeneration attributable to expression of neurofilament light chain (NF-L) transgenes have raised the possibility that the neuropathic effects result from overexpression of NF-L mRNA, independent of NF-L protein effects (Cañete-Soler et al., 1999). The present study was undertaken to test for an RNA-mediated pathogenesis. Transgenic mice were derived using either an enhanced green fluorescent protein reporter construct or modified chimeric constructs that differ only in their $3^{\prime}$ untranslated regions (UTRs). Motor function and spinal cord histology were normal in mice expressing the unmodified reporter transgene. In mice expressing a chimeric transgene in which sequence of NF-L $3^{\prime}$ UTR was inserted into the $3^{\prime}$ UTR of the reporter transgene, we
\end{abstract}

Neuropathic effects of neurofilament (NF) transgenes on motor neurons of transgenic mice were initially reported arising from high-level expressions of coding region and $3^{\prime}$ untranslated region (UTR) of a mouse NF-L (Xu et al., 1993) or human NF-H (Cote et al., 1993) transgene. A more severe form of motor neuron degeneration was subsequently reported in mice expressing low levels of a mutant NF-L transgene with a leucine-to-proline point mutation in the rod domain of the protein and a c-myc mutation at the end of the coding region (Lee et al., 1994). The point mutation was intended to produce a dominant disassembling subunit (Gill et al., 1990); however, expression of mutant protein caused accumulation rather than disassembly of NFs in degenerating motor neurons (Lee et al., 1994). Whether NF accumulations are the cause or result of motor neuron degeneration is still an unresolved issue.

An alternative view on the nature of NF-induced motor neuron degeneration arose from the discovery of a destabilizing element at the proximal edge of the NF-L mRNA 3' UTR (Cañete-Soler et al., 1998a). The location of the destabilizing element raised the possibility that marking the transgene with a $36 \mathrm{bp} c$-myc tag at the end of the coding region (Lee et al., 1994) may have conferred a second, possible neuropathic, mutation in the transgene. Insertion of the c-myc tag altered the stability of the transcript as well as the composition of RNA-binding complexes that assemble on the transcript (Cañete-Soler et al., 1999). Moreover, expression of a transgene with only the c-myc mutation has profound neuropathic effects on motor neurons of transgenic mice (Cañete-Soler et al.,

\footnotetext{
Received March 20, 2002; revised May 31, 2002; accepted June 21, 2002.

This study was supported by National Institutes of Health Grant NS35572.

Correspondence should be addressed to William W. Schlaepfer, 609C Stellar Chance Laboratories, University of Pennsylvania Medical School, Philadelphia, PA 19104-6100. E-mail: wws435jp@mail.med.upenn.edu.

Copyright (C) 2002 Society for Neuroscience $0270-6474 / 02 / 227662-09 \$ 15.00 / 0$
}

observed growth retardation and reduced kinetic activity during postnatal development. Older mice developed impairment of motor function and atrophy of nerve fibers in the ventral roots. A similar but more severe phenotype was observed when the chimeric transgene contained a 36 bp c-myc insert in an mRNA destabilizing element of the NF-L sequence. Our results suggest that neuropathic effects of overexpressing NF-L can occur at the level of transgene RNA and are mediated by sequences in the NF-L $3^{\prime}$ UTR.

Key words: RNA-mediated; neurofilament-induced; motor neuron degeneration; transgenic mice; EGFP reporter transgene; neuropathic RNA element

1999). The findings raise the possibility that RNA expression could have adverse effects on neurons, similar to the adverse effect of a mutant RNA on muscle of transgenic mice (Mankodi et al., 2000).

The presence of a neuropathic element in NF-L mRNA is still a unresolved issue. It could be argued that neuropathic effects of a c-myc mutation in the coding region of an NF-L transgene (Cañete-Soler et al., 1999) could arise from expression of mutant protein. The mutant protein could be disruptive by introducing a novel property or by altering interactions of endogenous NF-L protein. A dysfunctional state in motor neurons could also arise from a direct disruptive interaction of the mutant protein. It is even conceivable that expression of a mutant NF-L protein in an NF transgene could be disruptive by altering expression of endogenous NF gene or other gene products in motor neurons.

Establishing the level of gene expression leading to motor neuron degeneration by an NF transgene is essential before assessing any underlying mechanisms. Moreover, the presence of a neuropathic element in NF-L RNA is a novel and unexpected interpretation with far-reaching consequences. For these reasons, the present study set out to test for the presence of an untranslated neuropathic element in the NF-L transgene. NF-L sequences were placed in the $3^{\prime}$ UTR of an enhanced green fluorescent protein (EGFP) reporter gene and the chimeric reporter genes incorporated into the genome of transgenic mice. We found that expression of chimeric transgenes led to a dose-dependent impairment of motor function and that neuropathic elements are present in both wild-type (wt) and mutant NF-L mRNA. The findings have important implications for the pathogenesis of neurodegenerative disorders involving motor neurons and, possibly, other populations of neurons.

\section{MATERIALS AND METHODS}

Construction of transgenes and generation of transgenic mice. A $680 \mathrm{nt}$ fragment from $+1482 /+2161$ of mouse NF-L cDNA (Cañete-Soler et al., 
1998b) was inserted into the HindIII/BamH1 sites of pEGFP/C1 (Clontech, Palo Alto, CA) to construct the EGFP/NF-L transgene. NF-L sequence was amplified by PCR using 5'-TTCAAGCCTAGTAAGCTGAGGAGGCCAAGGAT-3' and 5'-TTCGGATCCTTTATTTACTATTTATTGCAC $-3^{\prime}$ as sense and antisense primers, thereby placing two stop codons (underlined) between the HindIII site (boldface) and the NF-L sequence. The same strategy was used to insert the identical NF-L sequence with 36 nt c-myc insert (Cañete-Soler et al., 1999) into $\mathrm{pEGFP} / \mathrm{C} 1$ as the EGFP/NF-L/c-myc transgene. Stop codon sequence (TAGTAA) was also placed behind the HindIII site of pEGFP/C1 for constructing the EGFP/wt transgene. The integrity of NF-L sequence, c-myc insert, and stop codons was confirmed by sequencing all final constructs.

The EGFP/NF-L, EGFP/NF-L/c-myc, and EGFP/wt constructs were excised from modified $\mathrm{pEGFP} / \mathrm{C} 1$ vectors with $A s e \mathrm{I}$ and $M l u \mathrm{I}$ and microinjected into fertilized eggs of B6SJF1/J $\times$ B6SJF1/J mice. Transgenic mice were identified by PCR and Southern blot of genomic DNA. Sense $(+842 /+862)$ and antisense $(+1296 /+1318)$ primers (see Fig. $1 A, A$ and $B)$ were used to amplify a $476 \mathrm{bp}$ PCR product from the transgene and to generate a radioactive cDNA probe $\left(10^{9} \mathrm{dpm} / \mu \mathrm{g}\right)$ for detecting a 756 bp transgenic fragment in Southern blots of genomic DNA digested with NheI and XhoI.

Transgene expression in transfected cells and transgenic mice. COS cells were transfected separately with the EGFP/NF-L, EGFP/NF-L/c-myc, or EGFP/wt constructs. Total RNA (120 ng) was isolated from transfected cells and used as templates for reverse transcription (RT)-PCR. PCR products were generated using a sense primer C $(+1214 /+1231)$ with either antisense primer D $(+1444 /+1424)$ to SV40 sequence or antisense primer E ( $5^{\prime}$-ACAGGCTGGCAGCAAGCCAGAAAGC- $\left.3^{\prime}\right)$ to NF-L sequence in the $3^{\prime}$ UTR of the transgenes (see Fig. $1 A$ ). Expression of EGFP protein was examined by immunoblotting lysates of transfected cells $(6 \mu \mathrm{g})$ with Living Color anti-EGFP antibody (Clontech).

Transgenes were introduced and propagated in B6SJLF1 mice. To assess transgene expression, a small drop of blood (1-2 $\mu \mathrm{l})$ was milked from the cut tip of mouse tails and dispersed in $500 \mu$ l of buffer (PBS with $2 \%$ calf serum and $1 \%$ formalin). Tails of 2 - to 3 -week-old mouse pups were presoaked in warm water to induce hyperemia. High levels of EGFP expression could be visualized directly by fluorescent microscopy. Immunohistochemical preparations of blood smears were treated with successive 15 min immersions in $1 \%$ hydrogen peroxide, $1 \%$ Triton $\mathrm{X}-100$, and normal goat serum and then incubated overnight at $4^{\circ} \mathrm{C}$ in 1:200, 1:500, 1:1000, and 1:2000 dilutions of Living Color anti-EGFP antibody (Clontech). Immunoreactivity was detected using a biotinylated secondary antibody, ABC reagents, and VIP substrate (Vector Laboratories, Burlingame, CA). Final preparations were counterstained with methyl green.

Generation of transgenic lines and assessment of phenotypic change. Transgenic mice expressing EGFP/NF-L, EGFP/NF-L/c-myc, and EGFP/wt transgenes were compared with nontransgenic littermates, together comprising over 1200 mice. Newborn litters were inspected for abnormal pups or perinatal demise, and the growth and development of viable pups were monitored daily. Tail clippings and blood samples were obtained at 2 weeks. Fo mice bearing transgenic DNA or expressing EGFP were retested at 3 weeks. Mice expressing high-level EGFP/NF-L or EGFP/wt transgene were bred to maximize transgene expression in the respective progeny. Transgenic and nontransgenic pups of transgenic lines were weighed and bled at $2,3,4,6$, and 8 weeks.

Kinetic activities and motor functions of mice expressing the EGFP/ NF-L transgene were compared with nontransgenic littermates or agematched mice expressing the EGFP/wt transgene. Tail-suspension tests were conducted to assess the ability of transgenic mice to exhibit normal writhing movements with asymmetrical extension of forelimbs and hindlimbs while reaching for proximal surfaces. A rotarod test was used to test motor coordination and balance. The rotarod apparatus consisted of a horizontal $18 \mathrm{~mm}$ round wooden dowel rotating at $10 \mathrm{rpm}$. Mice were habituated to the rotarod for a $5 \mathrm{~min}$ period of training before testing. Each test consisted of three consecutive attempts to remain on the rotarod for $60 \mathrm{sec}$ intervals. Retention times of EGFP/NF-L $(n=9)$ and $\mathrm{EGFP} / \mathrm{wt}(n=9)$ transgenic mice were averaged. Test 2 was conducted 1 week later.

Western blots of transgenic mouse tissues. Tissues of EGFP/NF-L transgenic mice exhibiting postnatal retarded growth or age-dependent motor impairment were divided so that the same tissue could be fixed by immersion in $4 \%$ paraformaldehyde or homogenized in $0.5 \%$ SDS, $8 \mathrm{M}$ urea, and $2 \% \beta$-mercaptoethanol. Tissues from age-matched EGFP/wt transgenic and nontransgenic mice were processed in the same manner. Fixed tissues of transgenic mice were processed for immunochemical assessment of EGFP expression (see below). Homogenized tissues were clarified by centrifugation, and supernatants were aliquoted and stored at $-80^{\circ} \mathrm{C}$. Tissues of transgenic mice showing anti-EGFP immunoreactivity in motor neurons were pooled for Western blot analyses. Duplicate samples of brain, spinal cord, dorsal root ganglia, and motor nerve roots were immunoblotted with the following primary NF antibodies: NF-L (NR4; Sigma, St. Lois, MO), NF-M (NN18; Sigma), NF-H-P+ (SMI31; Sternberger Monoclonals, Lutherville, MD), and NF-H-P- (SMI32; Sternberger Monolconals). Duplicate strips were also immunoblotted with anti-actin (sc-1616; Santa Cruz Biotechnology, Santa Cruz, CA). Biotin-conjugated primary antibodies were reacted with streptavidinperoxidase (Dako, Carpinteria, CA), and immune complexes were detected using lumi-light ${ }^{\text {plus }}$ Western blotting substrate (Roche Diagnostics, Indianopolis, IN). Similar studies compared NF expression in transgenic and nontransgenic mice.

Light microscopic and immunohistochemical analyses. Transgenic mice were anesthetized with $\mathrm{CO}_{2}$ and cardiac perfused with $10 \mathrm{ml}$ of PBS, followed by $20 \mathrm{ml}$ of PBS containing $4 \%$ paraformaldehyde. Neural and non-neural tissues were excised and immersed overnight in $4 \%$ paraformaldehyde, washed in PBS, dehydrated, and stored in $70 \%$ ethanol. Representative neural and non-neural tissues were embedded in paraffin. Spinal cords were cut and positioned in paraffin blocks to obtain representative cross-sections from cervical to lumbar cord (Cañete-Soler et al., 1999). Paraffin sections were stained with hematoxylin and eosin (H \& E) and immunoreacted for EGFP. Spinal cord sections of mice with highlevel expression of EGFP/NF-L and EGFP/wt transgenes were immunostained for NF proteins with the same battery of antibodies used in Western blots (see above). Immunoreactivities were detected using Vectastain Elite ABC (Vector Laboratories) and Dako ARK (Dako). Neural tissues were also immunoreacted for choline acetylase (Chemicon, Temecula, CA), GFAP (Sigma), and ubiquitin (Dako).

L4 and L5 nerve roots from transgenic mice were identified in fixed specimen of spinal cord during separation from vertebral columns. Nerve roots were excised, washed in phosphate buffer, and postfixed in Dalton's solution (1\% potassium dichromate and $2 \%$ osmium tetroxide in $0.85 \%$ $\mathrm{NaCl}$ ). Semithin cross-sections of dehydrated nerve were embedded and positioned in araldite 502 to facilitate transverse sectioning of nerve roots. One micrometer sections were stained with toluidine blue and visualized by phase microscopy. Analyses of myelinated fiber populations were performed on a Macintosh computer (Apple Computers, Cupertino, CA) using NIH Image (http://rsb.info.nih.gov/nih-image/). Software was programmed to enumerate the total number of myelinated nerve fibers and fiber size distribution by averaging maximum and minimum diameters.

\section{RESULTS}

\section{Generation of mice expressing an EGFP reporter gene with and without untranslated NF-L sequence in the 3' UTR}

NF-L sequence was placed in the $3^{\prime}$ UTR of an EGFP reporter transgene. Experimental (EGFP/NF-L and EGFP/NF-L/c-myc) and control (EGFP/wt) transgenes contained identical cDNA encoding EGFP behind a strong cytomegalovirus (CMV) promoter (Fig. 1A). The 3' UTRs contained SV40 early mRNA polyadenylation signaling sequence by itself (EGFP/wt), downstream of unaltered NF-L sequence (EGFP/NF-L) or downstream of NF-L sequence with a 36 nt c-myc insertion (EGFP/ NF-L/c-myc). NF-L sequence encompassed the entire $3^{\prime}$ UTR and adjacent coding region of mouse NF-L and included binding sites of multiple trans-acting factors (Cañete-Soler et al., 1998b). Stop codons were inserted at junctional sites of the chimeric constructs to ensure that NF elements remained untranslated. Transfections were undertaken to verify that NF elements in the chimeric transgenes were transcribed but not translated (Fig. $1 B, C)$.

Microinjections of transgenes into fertilized eggs generated five EGFP/wt, eight EGFP/NF-L, and four EGFP/NF-L/c-myc Fo 

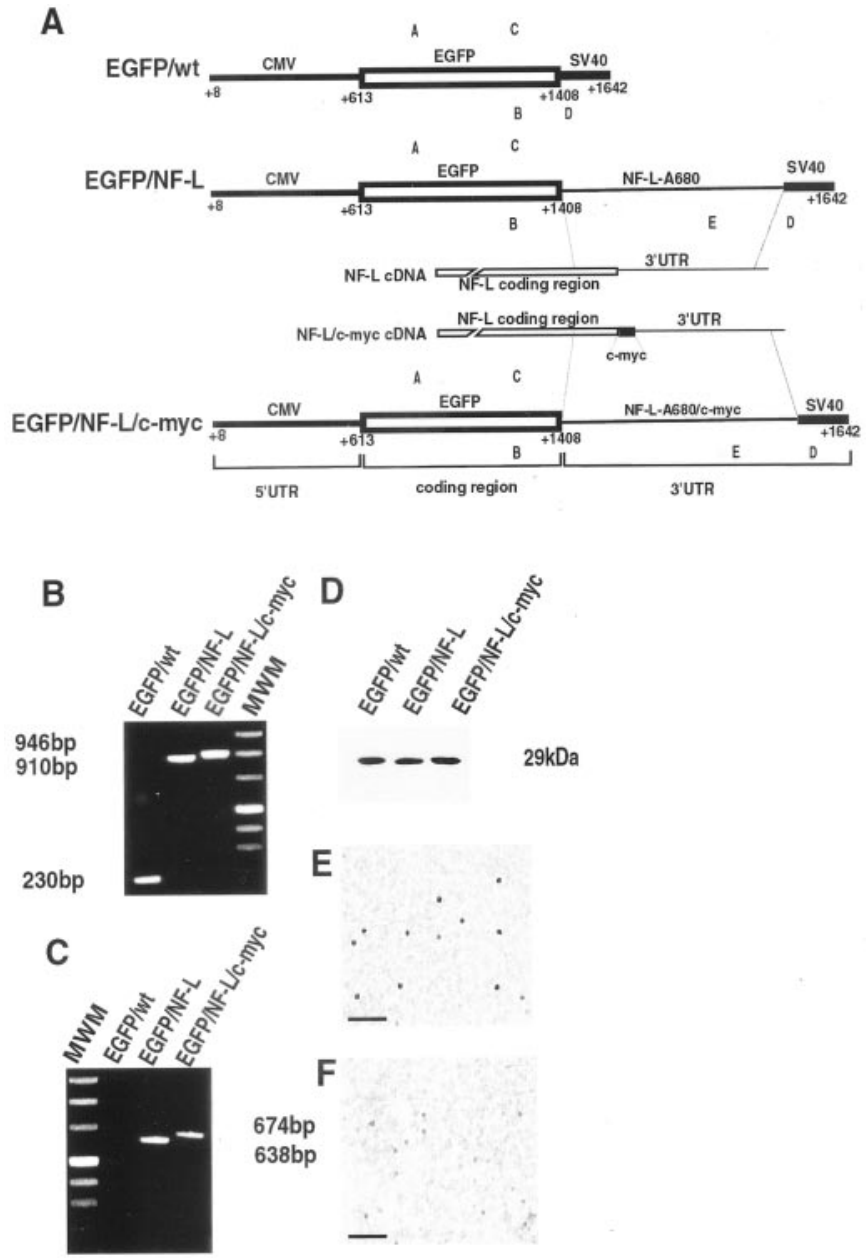

Figure 1. Construction of transgenes with NF-L sequence in $3^{\prime}$ UTR. $A$, Schematic diagram of the $5^{\prime}$ UTR coding region and $3^{\prime}$ UTR of the EGFP/wt, EFGP/NF-L, and EGFP/NF-L/c-myc transgenes. Each transgene contains a CMV promoter $(+8 /+613)$ in the $5^{\prime}$ UTRs, EGFP cDNA $(+613 /+1408)$ in the coding region, and SV40 polyadenylation sequence $(+1408 /+1642)$ in the $3^{\prime}$ UTR. A $680 \mathrm{nt}$ segment of mouse NF-L cDNA (NF-L-A680) was inserted into the proximal 3' UTR of EGFP/NF-L, and the same sequence with a $36 \mathrm{nt}$ c-myc tag (NF-L-A680/c-myc) was inserted into the proximal 3' UTR of EGFP/NF-L/c-myc. Nucleotide numbering is from the parent $\mathrm{pEGFP-C1}$ vector (Clontech). Locations of primers for RT-PCR are also shown. $B, C$, RT-PCR with primers A and $\mathrm{D}(B)$ or primers $\mathrm{C}$ and $\mathrm{E}(C)$ amplify RNA products of expected size in COS cells transfected with EFGP/wt, EGFP/NF-L, and EGFP/NF-L/cmyc transgenes. $D$, Anti-EGFP immunoblot of lysates from transfected COS cells showing expression of EGFP protein $(29 \mathrm{kDa})$ from $\mathrm{EFGP} / \mathrm{wt}$, EGFP/NF-L, and EGFP/NF-L/c-myc constructs. $E, F$, Detection of antiEGFP immunoreactivity in blood smears from an EGFP/NF-L transgenic pup $(E)$ but not from a nontransgenic littermate $(F)$. Scale bars, $100 \mu \mathrm{m}$. $M W M$, Molecular weight markers.

mice of a total of 48, 108, and 154 pups, respectively. An additional four EGFP/NF-L/c-myc pups died shortly after birth (0-2 d). The dead pups did not reveal an obvious phenotype. Their tissues were, however, incompletely recovered and were insufficiently preserved for neuropathological examination. A similar high incidence of perinatal deaths was also observed among transgenic pups bearing an NF-L transgene with the same c-myc mutation (Cañete-Soler et al., 1999).

Transgene expression was assessed in blood samples of transgenic mice (Fig. 1D,E), using serial dilutions of anti-EGFP antibody for semiquantitative analyses. Pups with high-level trans-
A
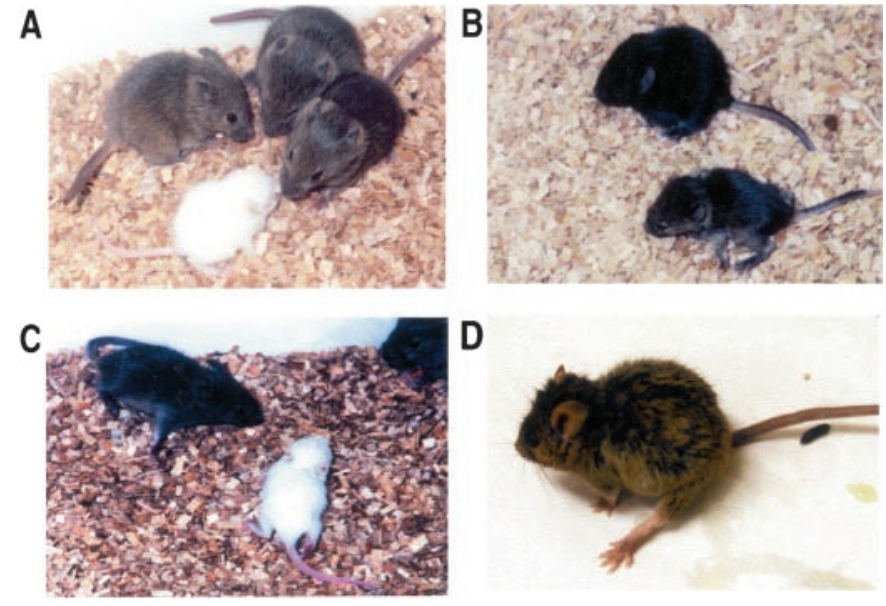

Figure 2. Retarded growth and impaired kinetic activity in EGFP/NF-L transgenic pups. $A$, Reduced size of a white EGFP/NF-L transgenic Fo pup compared with three nontransgenic agouti littermates at $18 \mathrm{~d}$. $B$, Small EGFP/NF-L transgenic Fo pup showing awkward positioning of hindlimbs and forelimbs in front of a nontransgenic littermate at $16 \mathrm{~d}$. $C$, Small white EGFP/NF-L transgenic F1 pup with nontransgenic agouti littermates at 18 d. $D$, EGFP/NF-L transgenic F1 pup with awkward extension of hindlimb and distal phalanges.

gene expression were bred. A total of 15 and six transgenic lines expressed EGFP/NF-L and EGFP/wt transgenes. Studies were directed at four lines expressing high levels of EGFP/NF-L and three lines expressing comparable levels of EGFP/wt. Founder mice bearing the EGFP/NF-L/c-myc construct expressed only limited amounts of the transgene, and expression of the transgene was further reduced during transmission of the transgenes in transgenic lines (see below).

\section{Expression of EGFP/NF-L transgene leads to retarded growth and reduced kinetic activity}

All Fo pups expressing the EGFP/NF-L transgene were smaller and less mobile than nontransgenic littermates (Fig. 2A,B). Severely affected pups moved reluctantly and only for short distances, often with asymmetrically extended limbs. These traits were most apparent at 2 and 3 weeks of age in Fo pups with high levels of EGFP/NF-L transgene expression. The EGFP/NF-L Fo pups were monitored carefully with the intent of killing the mice before their demise. However, the phenotype did not progress but receded and gradually disappeared during the 4-8 week period of development. Fo pups expressing EGFP/wt transgene could not be distinguished from nontransgenic littermates.

To assess retarded growth in transgenic lines, F1 (and F2) transgenic pups were weighed, examined, and monitored for EGFP expression in blood samples at 2, 3, 4, 6, and 8 weeks of age. Parallel studies were conducted on F1 (and F2) pups of EGFP/wt transgenic lines. Whereas similar levels of EGFP were observed in blood samples from EGFP/NF-L and EGFP/wt pups at 2 and 3 weeks of age, a progressive decline of EGFP expression was observed in EGFP/NF-L pups at 4, 6, and 8 weeks.

F1 and F2 pups from the four highest-expressing EGFP/NF-L transgenic lines exhibited dose-dependent retardation of growth and kinetic activity. Retarded growth was also observed in highexpressing F1 pups in other EGFP/NF-L transgenic lines. Maximum weight differential was observed at 2 weeks (Table 1). Weights of severely affected pups were $<50 \%$ of nontransgenic littermates. Recovery was also dose dependent. Averaged weights 


Table 1. Postnatal retardation in weights of EGFP/NF-L mice
\begin{tabular}{llllll} 
Transgene & EGFP expression $^{a}$ & $n$ & 2 weeks $^{b}$ & 3 weeks $^{b}$ & 4 weeks $^{b}$ \\
\hline None & & 114 & $6.8 \pm 1.4$ & $8.8 \pm 1.2$ & $12.4 \pm 2.4$ \\
EGFP/wt & $+/++/+++$ & 80 & $6.8 \pm 1.9^{* *}$ & $8.7 \pm 1.6^{*}$ & $12.4 \pm 2.5$ \\
EGFP/NF-L & $+/++$ & 71 & $5.8 \pm 0.6^{* *}$ & $8.1 \pm 1.0^{*}$ & $11.9 \pm 1.5$ \\
EGFP/NF-L & +++ & 34 & $4.6 \pm 0.8^{* *}$ & $6.7 \pm 1.8^{*}$ & $11.3 \pm 1.1$ \\
\hline
\end{tabular}

${ }^{a}$ Low $(+)$, moderate $(++)$, or high $(+++)$ levels of EGFP expression in blood.

${ }^{b}$ Weight measurements in grams.

${ }^{*} p<0.01 ;{ }^{* *} p<0.001$

of pups recovered between 2 and 4 weeks, but recovery was more prolonged in severely affected pups. Parallel studies on F1 and F2 transgenic pups expressing high levels of EGFP/wt transgene revealed similar weights to those of nontransgenic pups during the same period of postnatal development.

F1 and F2 pups with high levels of EGFP/NF-L expression also exhibited reduced mobility and abnormal movements of EGFP/ NF-L Fo pups (Fig. 2C,D). The affected pups were less responsive and moved for only short distances when prodded. They were readily captured by securing and lifting their tails. When suspended, they struggled less than age-matched controls and tended to flex rather than extend their limbs.

The EGFP/NF-L phenotype in transgenic lines was also most pronounced at 2 and 3 weeks and receded during the 4-8 week period of development, coinciding with reduction of transgene expression in blood. Parallel reductions in EGFP/NF-L expression and phenotype were also evidenced in successive F1, F2, and F3 generations of germ line transmission and in successive litters of Fo (and F1) breeders. A similar decline of transgene expression was not observed in EGFP/wt transgenic lines.

\section{EGFP/NF-L transgenic mice develop age-dependent impairment of motor function}

Mice bearing the EGFP/NF-L transgene developed an agedependent, slowly progressive deterioration of motor function. Age-dependent motor impairment was observed in six Fo mice and in $10 \mathrm{~F} 1$ mice from the four high-expressing transgenic lines. Age-dependent motor impairment occurred in Fo and F1 mice expressing high levels of EGFP/NF-L and exhibiting retarded growth and kinetic activity during postnatal development. Adultonset motor impairment was initially observed at 6-8 months and became pronounced at 12-16 months. Affected mice displayed an arched posture and waddling gait, using proximal musculature for locomotion to compensate for limited movements of distal hindlimb phalanges (Fig. $3 A$ ). End-stage weakness was evidenced by an inability to elevate during locomotion (Fig. $3 B$ ). When suspended, they flexed, instead of extending, their limbs and did not struggle or reach for proximal surfaces (Fig. $3 C$ ). When placed on a rotarod, they were unable to spread their hindlimb phalanges for the maintenance of balance. Instead, their hindlimb phalanges remained in a fixed, flexed and pronated position (Fig. 3D).

To quantitate motor impairment, a rotarod balancing test was conducted. Nontransgenic mice at 12-16 months were tested to establish baseline retention time at varying rotarod rotational speeds. Performance of EGFP/wt transgenic mice did not differ from the rotarod retention times of nontransgenic controls. However, age-matched EGFP/NF-L transgenic mice were markedly impaired in their ability to remain on the rotarod. Tests were therefore conducted at a relatively slow rotarod speed. Nevertheless, EGFP/NF-L transgenic mice with age-dependent motor
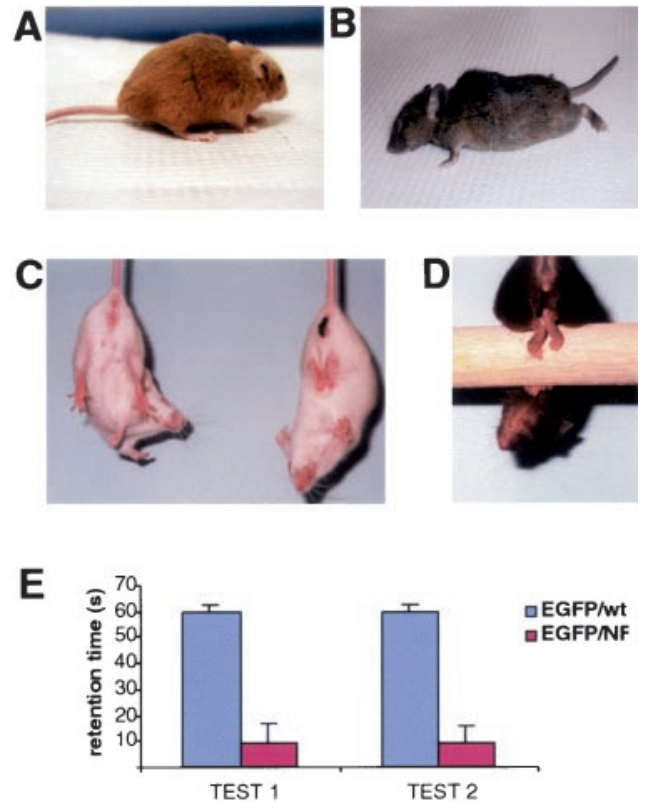

Figure 3. Age-dependent impairment of motor function of EGFP/NF-L transgenic mice. $A$, EGFP/NF-L F1 transgenic mouse at 12 months showing arched posture with hindlimb extension and widening of base. $B$, EGFP/NF-L Fo mouse at 18 months showing inability to elevate during locomotion. $C$, Abnormal limb flexion of EGFP/NF-L F1 mouse (right) compared with age-matched EGFP/wt mouse (left) during tail-suspension test. $D, \mathrm{EGFP} / \mathrm{NF}-\mathrm{L}$ F1 mouse with flexed and pronated phalanges unable to grasp rotarod surface. $E$, Results of rotarod performance tests of 12- to 16-month-old transgenic mice showing reduced retention times of EGFP/ NF-L transgenic mice. Tests 1 and 2 were conducted at $7 \mathrm{~d}$ intervals.

impairment were unable to balance themselves on the rotarod and did not improve their performance with practice or when retested 1 week later (Fig. $3 E$ ).

\section{Transgene expression does not alter NF expression in motor neurons}

Transgenic mice bearing EGFP/NF-L and EGFP/wt transgenes were killed by cardiac perfusion at varying intervals between 2 weeks and 18 months. EGFP immunoreactivity was widely distributed in neural and non-neural tissues during postnatal development. Similar levels were observed in EGFP/NF-L and EGFP/wt pups. However, an age-dependent reduction of EGFP expression was observed in non-neuronal tissues of EGFP/NF-L mice, to a lesser extent than the reduction of EGFP expression in blood samples during postnatal development (see above). EGFP expression in EGFP/NF-L mice persisted in neural tissues, especially in large neurons of brainstem and spinal cord. In spinal cord, the highest levels of EGFP expression were consistently observed in motor neurons of both EGFP/NF-L and EGFP/wt transgenic mice (Fig. 4A,B). Lower levels of immunoreactivity were present in neurons of the intermediate and posterior horns with less in glia and ependyma.

Endogenous NF expression was compared by immunohistochemical and Western blot in EGFP/NF-L mice with agedependent motor impairment at 12-16 months, in age-matched EGFP/wt transgenic mice and in age-matched nontransgenic controls. Similar patterns of immunoreactivities were observed at 14 months in motor neurons of motor-impaired EGFP/NF-L and in unimpaired EGFP/wt transgenic mice when probed with a battery of antibodies to different epitopes on NF-L, NF-M, and NF-H subunits (Fig. 4). Similar levels of NF expression were also 


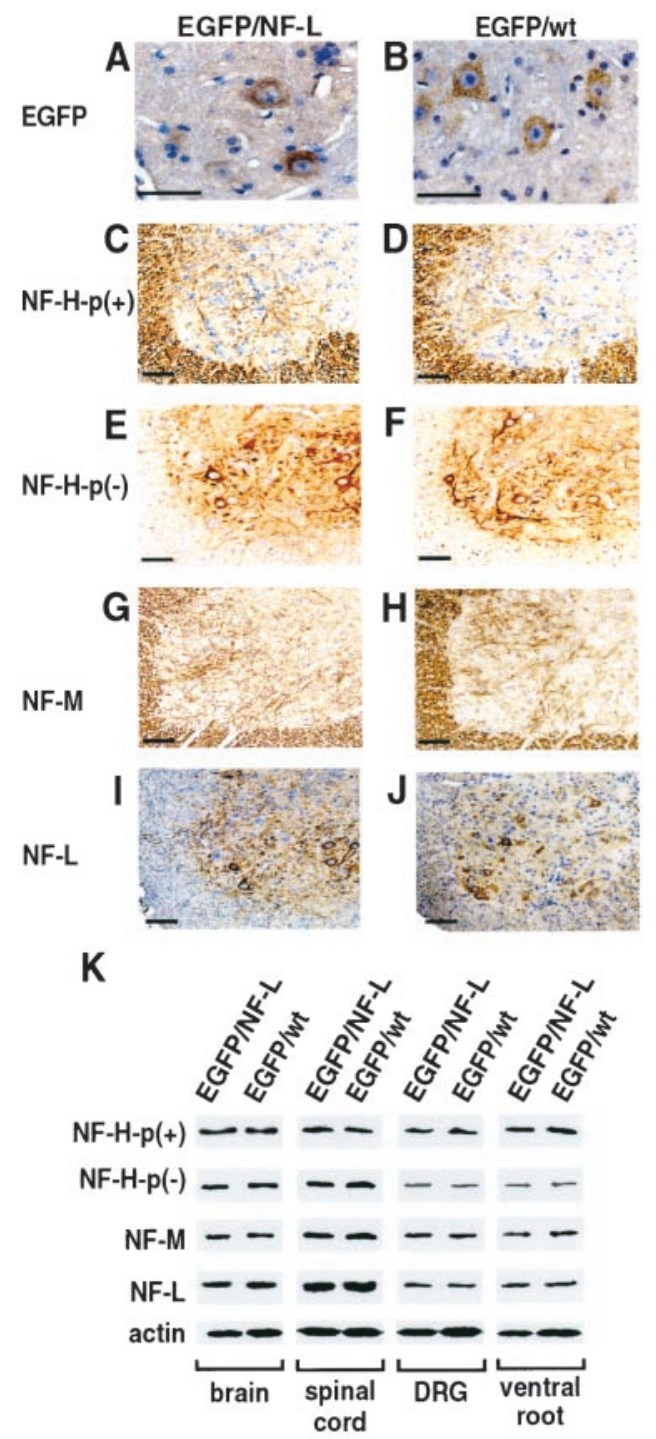

Figure 4. EGFP/NF-L phenotype is not associated with altered expression of NF proteins. $A-J$, Serial sections of motor neurons in lumbar spinal cords of 14-month-old EGFP/NF-L mouse with age-dependent motor impairment $(A, C, E, G, I)$ and of age-matched EGFP/wt mouse $(B, D, F, H, J)$ immunostained with antibodies to EGFP $(A, B)$, phosphorylated NF-H $(C, D)$, nonphosphorylated NF-H $(E, F)$, NF-M $(G$, $H)$, and NF-L $(I, J) . K$, Western blots of brain, spinal cord, dorsal root ganglia $(D R G)$, and spinal motor nerve roots of EGFP/NF-L and EGFP/wt transgenic mice immunoreacted with the same anti-NF antibodies. Anti-actin immunoreactivity was used as control. Tissues were pooled from age-matched mice showing anti-EGFP immunoreactivities in motor neurons. Scale bars, $100 \mu \mathrm{m}$.

observed in Western blots of brain, spinal cord, dorsal root ganglia, or spinal motor nerve roots of impaired EGFP/NF-L and unimpaired EGFP/wt transgenic mice at 12-16 months (Fig. $4 K$ ). Differences in NF expression could not be detected in tissues of transgenic mice during postnatal development or in mice with age-dependent motor impairment. Examination of systemic tissues of EGFP/NF-L mice did not reveal gross or microscopic alterations in the intestine to account for postnatal growth retardation.

\section{Age-dependent motor impairment in EGFP/NF-L mice associated with motor nerve fiber atrophy}

EGFP/NF-L transgenic mice with adult-onset motor impairment revealed a decrease of large myelinated fibers and relative in-
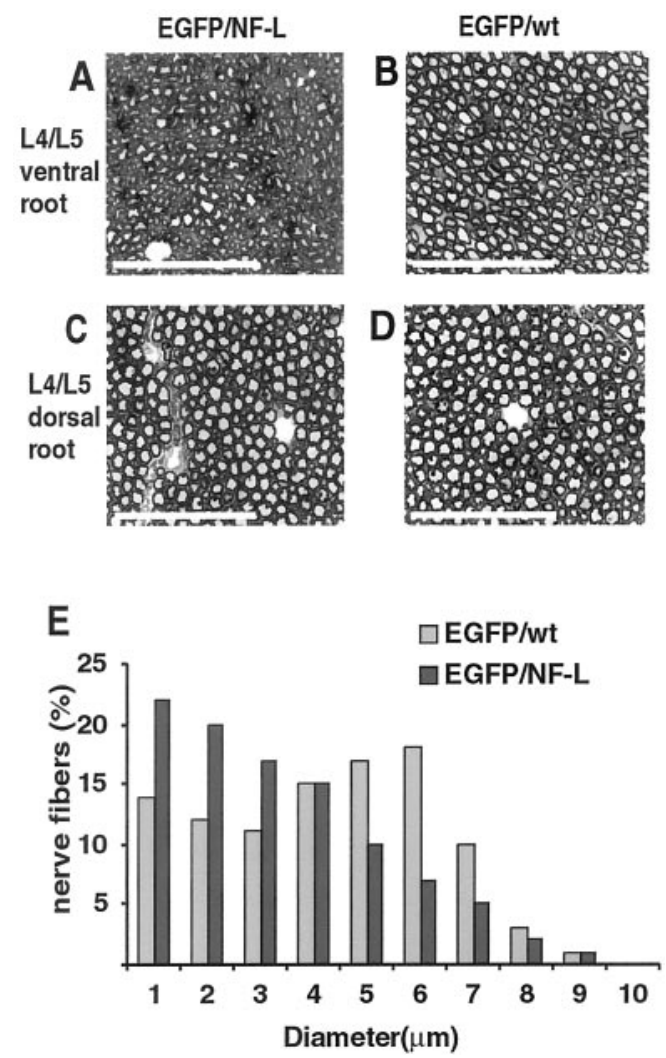

Figure 5. Atrophy of L4-L5 ventral spinal nerve roots in EGFP/NF-L transgenic mice with age-dependent motor impairment. $A-D$, Representative cross-section of $\mathrm{L} 4-\mathrm{L} 5$ ventral $(A, B)$ and dorsal $(C, D)$ spinal nerve roots from EGFP/NF-L $(A, C)$ and age-matched EGFP/wt $(B, D)$ transgenic mice showing loss of large myelinated fibers in motor roots, but not in sensory roots, of EGFP/NF-L transgenic mouse. Scale bars, 100 $\mu \mathrm{m}$. E, Quantitation of myelinated nerve fiber distribution in L4-L5 ventral nerve roots of EGFP/NF-L transgenic mice with agedependent motor impairment and age-matched EGFP/wt transgenic mice. Analyses were conducted on six sets of nerve roots from 12- to 16-month-old mice.

crease of small myelinated fibers in L4-L5 ventral roots compared with age-matched nontransgenic and EGFP/wt controls (Fig. 5A,B). The changes were specific for motor nerves because myelinated fibers in L4-L5 sensory roots were indistinguishable from those of age-matched controls (Fig. $5 C, D$ ). The relative increase of small myelinated fibers was not associated with a decline in the total numbers of myelinated nerve fibers in the L4-L5 ventral nerve roots.

The decrease in size of large nerve fibers in L4-L5 motor nerve roots was not accompanied by a corresponding measurable change in motor neurons of the lumbar spinal cord. No loss of motor neurons was observed, nor was reactive gliosis detected by anti-GFAP immunoreactivity. However, focal cytoplasmic vacuolization was observed in motor neurons of EGFP/NF-L transgenic mice with postnatal or age-dependent impairment of motor activity. Cytoplasmic vacuolization occurred in neurons with well preserved nuclear structure and often appeared as a loosening or dissolution of cytoplasmic structure (Fig. 6D). The cytoplasmic changes in motor neurons of EGFP/NF-L mice were deemed significant in view of their resemblance to severe vacuolar changes in motor neurons of mice expressing an EGFP/NF-L/cmyc transgene (see below). 

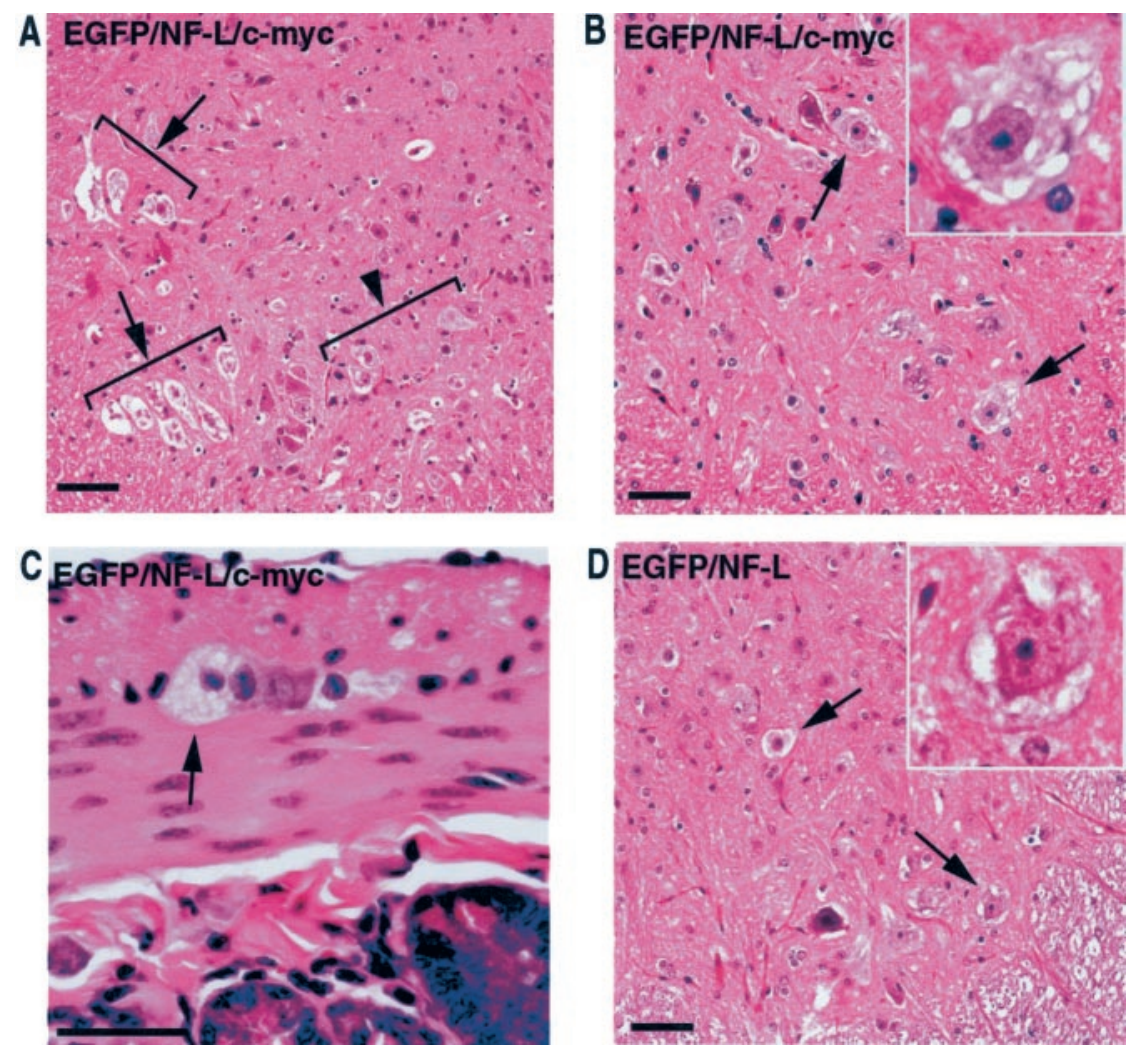

Figure 6. Cytoplasmic vacuolization in neurons of EGFP/NF-L/c-myc and EGFP/NF-L transgenic mice. $A$, Severe vacuolar changes in two lateral groups of motor neurons (arrows) and less severe changes in neurons of the medial anterior horn (arrowhead) in the lumbar spinal cord of C3 EGFP/NF-L/c-myc Fo mouse. $\mathrm{H} \&$ E-stained section. Scale bar, $100 \mu \mathrm{m} . \quad B$, Cytoplasmic vacuolar changes in motor neurons (arrows) of the cervical spinal cord of C3 EGFP/NF-L c-myc mouse, with inset showing enlargement of vacuolated neuron. $\mathrm{H}$ $\&$ E-stained section. Scale bar, $100 \mu \mathrm{m}$. C, Vacuolar changes in enteric neurons (arrow) of the myenteric plexus in the small intestine of C3 EGFP/NF-L/c-myc mouse. $\mathrm{H} \&$ E-stained section. Scale bar, $100 \mu \mathrm{m}$. $D$, Similar cytoplasmic vacuolar changes in motor neurons (arrows) in lumbar spinal cord of 16-month-old EGFP/ NF-L mouse with age-dependent impairment of motor activity and atrophy of L4-L5 motor nerve root fibers. Higher magnifications of vacuolated motor neuron is shown in the inset. H \& E-stained sections. Scale bar, $100 \mu \mathrm{m}$.
The c-myc mutation (EGFP/NF-L/c-myc) in the NF-L 3' UTR enhances the neuropathic effects of the chimeric transgene and leads to severe cytoplasmic vacuolization of motor neurons

Chimeric transgenes bearing the NF-L $3^{\prime}$ UTR with the c-myc mutation (EGFP/NF-L/c-myc) were poorly expressed in transgenic mice and could only be detected during postnatal development in blood cells of a single (C3) Fo female pup. Nevertheless, several Fo mice $(\mathrm{C} 2-\mathrm{C} 4)$ displayed transient postnatal growth retardation, similar to the EGFP/NF-L phenotype. At 2 months, C3 developed progressive weakness and paralysis and was killed during the early course of her first pregnancy. Microscopically, there was EGFP expression and extensive vacuolar degeneration of motor neurons throughout the spinal cord (Fig. 6A,B), in enteric neurons of the small intestine (Fig. $6 C$ ) and in groups of large neurons in the brainstem (data not shown). The vacuolar neuronal degeneration was the same as that produced by a mutant NF-L transgene with an identical c-myc mutation (Cañete-Soler et al., 1999).

The only male (C1) EGFP/NF-L/c-myc Fo mouse failed to transmit the transgene, whereas the $\mathrm{C} 2$ and $\mathrm{C} 4$ Fo mice were infertile. When killed at 9 months, $\mathrm{C} 1$ and $\mathrm{C} 2$ showed focal EGFP expression and vacuolar degenerative changes of motor neurons. An additional series of EGFP/NF-L/c-myc Fo mice were killed in utero during late embryonic development (embryonic day 19). They had a higher percentage of transgene transmission ( 5 of 48 pups) and were collectively smaller than their nontransgenic littermates but expressed only low levels of the transgene and revealed no obvious embryonic phenotype.

\section{DISCUSSION}

\section{Untranslated neuropathic element in NF-L transgene}

The placement of NF sequence in the 3' UTR of an EGFP reporter gene provides a transgenic model for testing neuropathic effects of untranslated sequence and simultaneously monitoring transgene expression. This strategy was used here to demonstrate the presence of an untranslated neuropathic element in mouse NF-L mRNA. Neuropathic effects resulted from expression of an EGFP reporter transgene containing untranslated NF-L sequence (EGFP/NF-L and EGFP/NF-L/c-myc) but not from a reporter transgene lacking NF-L sequence (EGFP/wt). The possibility of toxic effects of EGFP protein expression attributable to 3' UTR sequence cannot be totally excluded, although prolonged high-level expression of EGFP protein alone does not have deleterious effects on motor neurons of transgenic mice (Feng et al., 2000). Functional and structural changes of motor neurons correlated with levels of transgene expression in blood samples of live pups and with transgene expression in tissues, especially motor neurons, of transgenic mice. Moreover, the transfer of neuropathic effects to a heterologous gene product demonstrates a context-independent feature of the neuropathic element and suggests that expression of untranslated NF sequence per se is sufficient to confer adverse effects on a susceptible subset of neurons.

A neuropathic element in the NF-L transcript was initially suggested on the basis of the motor neuron degeneration caused by an NF-L transgene with a c-myc mutation in a destabilizing element of NF-L mRNA (Cañete-Soler et al., 1999). These findings raised the possibility that neuropathic effects may also arise from RNA with an unaltered destabilizing element in the $3^{\prime}$ UTR of the NF-L and NF-H transcripts (Cañete-Soler and Schlaepfer, 2000) to account for the motor neuron degeneration in mice overexpressing an NF-L (Xu et al., 1993) or NF-H (Cote et al., 1993) transgene. The present study now supports the presence of a neuropathic element in unaltered NF-L RNA and suggests that inserting a c-myc mutation into the destabilizing element of the transcript may enhance the neuropathic effects of NF-L RNA. 
The phenotype in mice expressing high levels of untranslated NF-L RNA closely resembled the phenotype reported in mice expressing high levels of an NF-L transgene (Xu et al., 1993). The latter phenotype, achieved by the crossbreeding of singly transgenic lines, was readily apparent by the reduction in size and kinetic activity of doubly transgenic pups. Originally described in eight pups (Xu et al., 1993), our analyses of over 100 transgenic pups showed that the phenotype is dose dependent. In both instances, the phenotype was readily observed at 2-3 weeks but was less apparent at $6-8$ weeks. The occurrence of the same phenotype from a transgene expressing untranslated NF-L sequence and from expression of the same sequence in the context of an NF-L transgene suggests that the phenotypes are causally related and not dependent on translation of the NF-L element.

It could be argued that neuropathic effects of untranslated NF-L sequence arise from alterations of endogenous NF gene expression with accumulation of NF protein in motor neurons. This possibility can be discounted on the grounds that expression of the EGFP/NF-L transgene did not alter NF expression by Western blots or by NF immunoreactivity in motor neurons, especially in view of the several-fold increases of endogenous NF expression associated with motor neuron degeneration (Cote et al., 1993; Xu et al., 1993). NF accumulations are known to arise from alterations in NF subunit stoichiometry and are the expected outcome from overexpression of a translatable NF-L transgene. Whether NF accumulation leads to the neuropathic phenotype is less clear. The present study suggests that the phenotype is attributable to expression of NF-L RNA and not NF-L protein or NF accumulations in motor neurons.

Vacuolar changes in motor neurons is a distinctive alteration in mice with a c-myc mutation in the destabilizing element of transgenic NF-L mRNA (Cañete-Soler et al., 1999). Similar changes were also depicted in motor neurons of mice expressing another NF-L transgene containing the same c-myc mutation (Lee et al., 1994, their Figs. 3B, F, 8B). The occurrence of these changes in the present study suggests that they arise from untranslated NF-L elements. The nature of the changes is, however, unclear because severe vacuolization is not associated with apparent neuronal loss or reactive gliosis. Profound neurological deficits may occur with limited structural alterations (Abel et al., 2001; Adachi et al., 2001), including phenotypes arising in mice bearing NF transgenes (Bruijn and Cleveland, 1996).

\section{RNA-binding proteins establish and maintain neuronal differentiation}

The identification of a neuropathic element in the NF-L transcript and the transfer of the neuropathic element to a heterologous transcript implicate RNA-binding proteins in the neurodegenerative state. Similar phenomena occur in myotonic dystrophy (Tapscott and Thornton, 2001) attributable to an expanded CTG repeat in the 3' UTR of the DMPK gene at the DM1 locus (Brook et al., 1992) or an expanded CCTG repeat in intron 1 of the ZNF9 gene at the DM2 locus (Liquori et al., 2001). Expression of the expanded CTG repeat on a heterologous gene reproduces the myotonic phenotype in transgenic mice (Mankodi et al., 2000). The mechanisms of a trans-active RNA-mediated pathology are, however, still poorly understood. Assemblage of RNA-binding proteins on a mutant, expanded, or overexpressed RNA sequence could effect their abilities to process other RNA substrates in the cell. Binding to an expanded CUG repeat in the DMPK mRNA alters the disposition and activity of cognate CUG-binding proteins (Timchenko et al., 2001). CUG-binding proteins comprise a large conserved family of RNA binding proteins, closely related to Elav/Hu with similar widespread and profound effects on the processing of RNA (Lu et al., 1999; Milne and Hodgkin, 1999; Good et al., 2000; Miller et al., 2000; Ladd et al., 2001).

The Elav/Hu family is a strongly conserved, predominantly neuronal set of RNA-binding proteins that bind adenosine and uridine-rich elements (ARE) (Good, 1995; Keene, 1999; Brennan and Steitz, 2001). HuR has evolved as a ubiquitously expressed member of the Elav/Hu family, whereas $\mathrm{HuB}, \mathrm{HuC}$, and $\mathrm{HuD}$ have remained primarily neuron specific and closely associated with neuronal differentiation (Akamatsu et al., 1999). Elav/Huinduced neuronal differentiation is accompanied by increased stability or translations of neuronal transcripts containing ARE (Antic et al., 1999; Aranda-Abreu et al., 1999; Mobarak et al., 2000). Overexpression of the ARE binding sites can have a trans-active effect in decreasing expression of endogenous GAP43 and preventing NGF-induce neuronal differentiation (Neve et al., 1999).

Expression of the prototype Elav protein is also necessary for maintaining the differentiated state of neurons in the retina of Drosophila (Campos et al., 1985; Yao et al., 1993). Flies bearing a temperature-sensitive mutant elav gene develop normally at permissive temperatures but undergo neuronal degeneration when shifted to a nonpermissive temperature (Homyk et al., 1985). Interestingly, similar phenomena occur from overexpression of an elav gene (elr-1) in motor neurons of Caenorhabditis elegans (Fujita et al., 1999). Heat-induced upregulation of elr-1 under control of a heat shock promoter produced a lethal phenotype in adult and late larval worms. Levels of elav expression in Drosophila are tightly autoregulated, and a highly deleterious condition arises when the autoregulatory element is deleted (Samson, 1998). Autoregulatory elements have been described in mouse $H u B$ (Abe et al., 1996).

Shifting pattern of $\mathrm{Hu}$ expression during neuronal differentiation in mouse (Okano and Darnell, 1997), chicken (Wakamatsu and Weston, 1997), Xenopus (Perron et al., 1999), and zebrafish (Park et al., 2000) suggests that RNA-binding proteins may have differing effects on differentiation in subsets of neurons. Persistent high-level expression of specific Hu proteins in subsets of differentiated neurons (Clayton et al., 1998) may indicate an additional functional role in maintaining neuronal phenotype. A role of Elav/Hu proteins in maintaining neuronal homeostasis is also suggested by the paraneoplastic neurological syndrome whereby autoantibodies to neuronal RNA-binding protein arise from neoplastic tissues and lead to selective degeneration of different subsets of neurons (Musunuru and Darnell, 2001).

The full identity of neuropathic elements in NF-L mRNA is still unclear. We suspect that neuropathic effects are mediated by RNA-binding proteins whose presence and disposition are modified by a c-myc mutation in a manner that accentuates the neuropathic effects on expression of the respective elements in motor neurons. Replacement of 3' UTR of NF-H by a lacZ gene deprives the transgenic mRNA of similar destabilizing element and could explain why an NF-H/lacZ chimeric transgene causes massive accumulation of NFs without progressive degeneration of motor neurons in transgenic mice (Eyer and Peterson, 1994).

\section{Specificity of RNA-mediated neuropathic effects}

Loss or mutation of an RNA-binding protein leads to motor neuron degeneration in classical (Liu et al., 1997) and variant (Grohmann et al., 2001) forms of spinal muscular atrophy and to neuronal degeneration in the fragile $\mathrm{X}$ syndrome (Siomi et al., 
1993). In each instance, subsets of neurons, including motor neurons, are selectively susceptible to alterations of a widely expressed RNA-binding protein. The neuropathic element in NF-L mRNA also has a highly selective neuropathic effect, although the transcript is widely expressed in neuronal tissues. Although the underlying mechanisms are not understood, the specificity of neuronal degeneration may be instructive. The findings suggest that specific RNA-binding proteins, cofactors, or target binding sites may have differential effects on gene expression in subsets of neurons.

Selective vulnerabilities of neurons to an alteration in an RNAbinding protein or RNA binding site presuppose a specificity in posttranscriptional regulation among different subsets of neurons. Such diversity in posttranscriptional pathways may have arisen during development of neuronal circuitries when vast numbers of neurons become trophic-factor dependent and require distinctive signals from specific target neurons for survival (Burek and Oppenheim, 1996). Neurons then lose their trophic factor dependency, possibly by altering signal transduction pathways (Mielke and Herdegen, 2000). Specificity of RNA-mediated neuropathic effects may arise out of inherent differences in the composition of posttranscriptional regulatory factors and their abilities to maintain homeostasis of different subsets of neurons.

Specificity of motor neuron degeneration has been an elusive issue in explaining the gain-in-adverse function attributable to a widely expressed mutant superoxide dismutase-1 (SOD-1) protein (Cleveland and Rothstein, 2001). The presence of a neuropathic element in the NF-L transcripts now raises the possibility that interactions with posttranscriptional regulatory factors may account for the reduced neuropathic effects of an SOD-1 transgene in mice either lacking an endogenous NF-L gene (Williamson et al., 1998) or overexpressing an NF-H transgene (CouillardDespres et al., 1998) but not in mice overexpressing an NF-H transgene lacking the mRNA destabilizing element in the $3^{\prime}$ UTR (Eyer et al., 1998). Although deletion of the NF-L gene reduces the toxic effects of a mutant SOD-1 transgene on motor neurons, it increases the neuropathic effects on primary sensory neurons (Williamson et al., 1998), suggesting a different but related homeostatic mechanism in different subsets of neurons. There is also a paradoxical reduction of mutant SOD-1-induced pathology in motor neurons after nerve transection (Kong and Xu, 1999), a procedure known to destabilize NF mRNAs and reduce levels of NF expression (Schwartz et al., 1992). Interactions with posttranscriptional factors could link the selective vulnerability of motor neurons to a mutant SOD-1 protein.

RNA-mediated motor neuron degeneration provides a model for probing linkage between a neuropathic element and neuronal homeostasis. Recent studies have identified p190RhoGEF as a brain-enriched, highly interactive exchange factor (Zhai et al., 2001) that binds to the destabilizing element in NF-L mRNA and stabilizes the transcript (Cañete-Soler et al., 2001). p190RhoGEF also binds c-Jun N-terminal kinase (JNK) interacting protein (JIP-1) (Meyer et al., 1999) and has anti-apoptotic activity, possibly by sequestering JIP-1 and JNK in the cytoplasm (CañeteSoler, unpublished data). Interestingly, another GEF protein was identified recently as the mutant gene product in a recessive form of familial motor neuron degeneration (Hadano et al., 2001; Yang et al., 2001). Further identification and characterization of transacting factors and their specific interactions in vulnerable subsets of neurons could provide important insights into the mechanisms of RNA-mediated neurodegeneration.

\section{REFERENCES}

Abe R, Yamamoto K, Sakamoto H (1996) Target specificity of neuronal RNA-binding protein, Mel-N1: direct binding to the $3^{\prime}$ untranslated region of its own mRNA. Nucleic Acids Res 24:2011-2016.

Abel A, Walcott J, Woods J, Duda J, Merry DE (2001) Expression of expanded repeat androgen receptor produces neurologic disease in transgenic mice. Hum Mol Genet 10:107-110.

Adachi H, Kume A, Li M, Nakagomi Y, Niwa H, Do J, Sang C, Kobayashi Y, Doyu M, Sobue G (2001) Transgenic mice with an expanded CAG repeat controlled by the human AR promoter show polyglutamine nuclear inclusions and neuronal dysfunction without neuronal cell death. Hum Mol Genet 10:1039-1048.

Akamatsu W, Okano HJ, Osumi N, Inoue T, Nakamura S, Sakakibara S, Miura M, Matsuo N, Darnell RB, Okano H (1999) Mammalian ELAV-like neuronal RNA-binding proteins $\mathrm{HuB}$ and HUC promote neuronal development in both the central and the peripheral nervous systems. Proc Natl Acad Sci USA 17:9885-9890.

Antic D, Lu N, Keene JD (1999) ELAV tumor antigen, Hel-N1, increases translation of neurofilament $\mathrm{M}$ mRNA and induces formation of neurites in human teratocarcinoma cells. Genes Dev 13:449-461.

Aranda-Abreu GE, Behar L, Chung S, Furneaux H, Ginzburg I (1999) Embryonic lethal abnormal vision-like RNA-binding proteins regulate neurite outgrowth and tau expression in PC12 cells. J Neurosci 19:6907-6917.

Brennan CM, Steitz JA (2001) HuR and mRNA stability. Cell Mol Life Sci 58:266-277.

Brook JD, McCurrach ME, Harley HG, Buckler AJ, Church D, Aburatani H, Hunter K, Stanton VP, Thirion JP, Hudson T (1992) Molecular basis of myotonic dystrophy: expansion of a trinucleotide (CTG) repeat at the $3^{\prime}$ end of the transcript encoding a protein kinase family member. Cell 68:799-808.

Bruijn LI, Cleveland DW (1996) Mechanisms of selective motor neuron death in ALS: insights from transgenic mouse models of motor neuron disease. Neuropath Appl Neurobiol 22:373-387.

Burek MJ, Oppenheim RW (1996) Programmed cell death in the developing nervous system. Brain Pathol 6:427-446.

Campos AR, Grossman D, White K (1985) Mutant alleles at the locus elav of Drosophila melanogaster lead to nervous system defects: a developmental-genetic analysis. J Neurogenet 2:197-218.

Cañete-Soler R, Schlaepfer WW (2000) Similar poly(C)-sensitive ribonucleoprotein complexes regulate the stability of the heavy and light neurofilament mRNAs. Brain Res 867:265-279.

Cañete-Soler R, Schwartz ML, Hua Y, Schlaepfer WW (1998a) Stability determinants are localized to the $3^{\prime}$ UTR and $3^{\prime}$ coding region of the neurofilament light subunit mRNA using a tetracycline-inducible promoter. J Biol Chem 273:12650-12654.

Cañete-Soler R, Schwartz ML, Hua Y, Schlaepfer WW (1998b) Characterization of ribonucleoprotein complexes and their binding sites on the neurofilament light subunit (NF-L) mRNA. J Biol Chem 273:12655-12661.

Cañete-Soler R, Silberg DG, Gershon MD, Schlaepfer WW (1999) Mutation in neurofilament transgene implicates RNA processing in the pathogenesis of neurodegenerative disease. J Neurosci 19:1273-1283.

Cañete-Soler R, Wu J, Zhai J, Shamim M, Schlaepfer WW (2001) p190RhoGEF binds to a destabilizing element in the 3'untranslated region of light neurofilament subunit mRNA and alters the stability of the transcript. J Biol Chem 276:32046-32050.

Clayton GH, Perez GM, Smith RL, Owen GC (1998) Expression of mRNA for the elav-like neuronal-specific RNA binding protein, HuD, during nervous system development. Brain Res Dev Brain Res 109:271-280.

Cleveland DW, Rothstein JD (2001) From Charcot to Lou Gehrig: deciphering selective motor neuron death in ALS. Nat Neurosci Rev 2:806-819.

Cote F, Collard J-F, Julien J-P (1993) Progressive neuronopathy in transgenic mice expressing the human neurofilament heavy gene: a mouse model of amyotrophic lateral sclerosis. Cell 73:35-46.

Couillard-Despres S, Z hu Q, Wong P, Price DL, Cleveland DW, Julien J-P (1998) Protective effect of neurofilament heavy gene overexpression in motor neuron disease induced by mutant superoxide dismutase. Proc Natl Acad Sci USA 95:9626-9630.

Eyer J, Peterson A (1994) Neurofilament-deficient axons and perikaryal aggregates in viable transgenic mice expressing a neurofilament- $\beta$ galactosidase fusion protein. Neuron 112:389-405.

Eyer J, Cleveland DW, Wong PC, Peterson AC (1998) Pathogenesis of two axonopathies does not require axonal neurofilaments. Nature 391:584-587.

Feng G, Mellor RH, Bernstein M, Keller-Peck C, Nguyen QT, Wallace M, Nerbonne JM, Lichtman JW, Sanes JR (2000) Imaging neuronal subsets in transgenic mice expressing multiple spectral variants of GFP. Neuron 28:41-51.

Fujita M, Kawano T, Ohta A, Sakamoto H (1999) Neuronal expression of a Caenorhabditis elegans elav-like gene and the effects of its ectopic expression. Biochem Biophys Res Commun 260:646-652. 
Gill SR, Wong PC, Monteiro MJ, Cleveland DW (1990) Assembly properties of dominant and recessive mutations in the small mouse neurofilament (NF-L) subunit. J Cell Biol 111:2005-2019.

Good PJ (1995) A conserved family of elav-like genes in vertebrates. Proc Natl Acad Sci USA 92:4557-4561.

Good PJ, Chen Q, Warner SJ, Herring DC (2000) A family of human RNA-binding proteins related to the Drosophila Bruno translational regulator. J Biol Chem 275:28583-28592.

Grohmann K, Schuelke M, Diers A, Hoffmann K, Lucke B, Adams C, Bertini E, Leonhardt-Horti H, Muntoni F, Ouvrier R, Pfeufer A, Rossi R, Van Maldergem L, Wilmshurs JM, Wienker TF, Sendtner M, Rudnik-Schoneborn S, Zerres K, Hubner C (2001) Mutations in the gene encoding immunoglobin $\mu$-binding protein 2 cause spinal muscular atrophy with respiratory distress type 1. Nat Genet 29:75-77.

Hadano S, Hand CK, Osuga H, Yanagisawa Y, Otomo A, Devon RS, Miyamoto N, Showguchi-Miyata J, Okada Y, Singaraja R, Figlewicz DA, Kwiatkowski T, Hosler BA, Sagie T, Skaug J, Nasir J, Brown RH, Scherer SW, Rouleau G, Hayden M, Ikeda J (2001) A gene encoding a putative GTPase regulator is mutated in familial amyotrophic lateral sclerosis 2. Nat Genet 29:166-173.

Homyk TJ, Isono K, Pak WL (1985) Developmental and physiological analysis of a conditional mutation affecting photoreceptor and lobe development in Drosophila melanogaster. J Neurogenet 2:309-324.

Keene JD (1999) Why is Hu where? Shuttling of early-response-gene messenger RNA subsets. Proc Natl Acad Sci USA 96:5-7.

Kong J, Xu Z (1999) Peripheral axotomy slows motoneuron degeneration in a transgenic mouse line expressing SOD1 G93A. J Comp Neurol 412:373-380.

Ladd AN, Charlet BN, Cooper TA (2001) The CELF family of RNA binding proteins is implicated in cell-specific and developmentally regulated alternative splicing. Mol Cell Biol 21:1285-1296.

Lee MK, Marszalek JR, Cleveland DW (1994) A mutant neurofilament subunit causes massive, selective motor neuron death: implications for the pathogenesis of human motor neuron disease. Neuron 13:975-988.

Liquori CL, Ricker K, Moseley ML, Jacobsen JF, Kress W, Naylor SL, Day JW, Ranum LPW (2001) Myotonic dystrophy type 2 caused by a CCTG expansion in intron 1 of ZNF9. Science 293:864-867.

Liu Q, Fischer U, Wang F, Dreyfuss G (1997) The spinal muscular atrophy disease gene product, SMN, and its associated protein SIP1 are in a complex with spliceosomal snRNP proteins. Cell 90:1013-1021.

Lu X, Timchenko NA, Timchenko LT (1999) Cardiac elav-type RNAbinding protein (ETR-3) binds to RNA CUG repeats expanded in myotonic dystrophy. Hum Mol Genet 8:53-60.

Mankodi A, Logigian E, Callahan L, McClain C, White R, Henderson D, Krym M, Thornton CA (2000) Myotonic dystrophy in transgenic mice expressing an expanded CUG repeat. Science 289:1769-1772.

Meyer D, Liu A, Margolis B (1999) Interaction of c-Jun amino-terminal kinase interacting protein-1 with p190RhoGEF and its localization in differentiated neurons. J Biol Chem 274:35113-35118.

Mielke K, Herdegen T (2000) JNK and p38 stress kinases-degenerative effectors of signal transduction cascades in the nervous system. Prog Neurobiol 61:45-60.

Miller JW, Urbinati CR, Teng-Umnuay P, Stenberg MG, Byrne BJ, Thornton CA, Swanson MS (2000) Recruitment of human muscleblind proteins to (CUG)n expansions associated with myotonic dystrophy. EMBO J 19:4439-4448

Milne CA, Hodgkin J (1999) ETR-1, a homologue of a protein linked to myotonic dystrophy, is essential for muscle development in Caenorhabditis elegans. Curr Biol 9:1243-1246.
Mobarak CD, Anderson KD, Morin M, Beckel-Mitchener A, Rogers SL, Furneaux H, King P, Perrone-Biozerozz NI (2000) The RNA-binding protein $\mathrm{HuD}$ is required for GAP-43 mRNA stability, GAP-43 gene expression, and PKC-dependent neurite outgrowth in PC12 cells. Mol Cell Biol 11:3191-3203.

Musunuru K, Darnell RB (2001) Paraneoplastic neurological disease antigens: RNA-binding proteins and signaling proteins in neuronal degeneration. Annu Rev Neurosci 24:239-262.

Neve RL, Ivins KJ, Tsai KC, Rogers SL, Perrone-Biozerozz NI (1999) Cis-acting regulatory elements in the GAP-43 mRNA $3^{\prime}$ untranslated region can function in trans to suppress endogenous GAP-43 gene expression. Brain Res Mol Brain Res 65:52-60.

Okano HJ, Darnell RB (1997) A hierarchy of Hu RNA binding proteins in developing and adult neurons. J Neurosci 17:3024-3037.

Park HC, Hong SK, Kim HS, Kim SH, Yoon EJ, Kim CH, Miki N, Huh TL (2000) Structural comparison of zebrafish Elav/Hu and their differential expressions during neurogenesis. Neurosci Lett 28:81-84.

Perron M, Furrer MP, Wegnez M, Theodore L (1999) Xenopus elav-like genes are differentially expressed during neurogenesis. Mech Dev 84:139-144.

Samson M-L (1998) Evidence for 3' untranslated region-dependent autoregulation of the Drosophila gene encoding the neuronal nuclear RNA-binding protein ELAV. Genetics 150:723-733.

Schwartz ML, Shneidman PS, Bruce J, Schlaepfer WW (1992) Actinomycin prevents the destabilization of neurofilament mRNA in primary sensory neurons. J Biol Chem 267:24596-24600.

Siomi H, Siomi MC, Nussbaum RL, Dreyfuss G (1993) The protein product of fragile $\mathrm{X}$ gene, FMR1, has characteristics of an RNAbinding protein. Cell 74:291-298.

Tapscott S, Thornton CA (2001) Reconstructing myotonic dystrophy. Science 293:816-817.

Timchenko NA, Cai Z-J, Welm AL, Reddy S, Ashizawa T, Timchenko LT (2001) RNA CUG repeats sequester CUGBP1 and alter protein levels and activity of CUGBP1. J Biol Chem 276:7820-7826.

Wakamatsu Y, Weston JA (1997) Sequential expression and role of $\mathrm{Hu}$ RNA-binding proteins during neurogenesis. Development 124:34493460 .

Williamson TL, Bruijn LI, Zhu Q, Anderson KL, Anderson SC, Julien J-P, Cleveland DW (1998) Absence of neurofilaments reduces the selective vulnerability of motor neurons and slows disease caused by a familial amyotrophic lateral sclerosis-linked superoxide dismutase 1 mutant. Proc Natl Acad Sci USA 95:9631-9636.

Xu Z, Cork LC, Griffin JW, Cleveland DW (1993) Increased expression of neurofilament subunit NF-L produces morphological alterations that resemble the pathology of human motor neuron disease. Cell 73:23-33.

Yang Y, Hentati A, Deng HX, Dabbagh O, Sasaki T, Hirano M, Hung WY, Ouahchi K, Yan J, Azim A, Cole N, Gascon G, Yagmour A, Ben-Hamida M, Pericak-Vance M, Hentati F, Siddique T (2001) The gene encoding alsin, a protein with three guanine-nucleotide exchange factor domains, is mutated in a form of recessive amyotrophic lateral sclerosis. Nat Genet 29:160-165.

Yao KM, Samson ML, Reeves R, White K (1993) Gene elav of Drosophila melanogaster: a prototype for neuronal-specific RNA binding protein gene family that is conserved in flies and humans. J Neurobiol 24:723-739.

Zhai J, Lin H, Shamim M, Schlaepfer WW, Cañete-Soler R (2001) Identification of a novel interaction of 14-3-3 with p190RhoGEF. J Biol Chem 276:41318-41324. 